\title{
Tuna, Mehmet H. (2020). Islamische Religionslehrer*innen auf dem Weg zur Professionalisierung (Studien zur islamischen Theologie und Religionspädagogik). Münster: Waxmann. ISBN 978-3-8309-4086-9. 3 I 5 Seiten.
}

\author{
Carina Caruso \\ Universität Paderborn (ccaruso@mail.uni-paderborn.de)
}

Fragen im Zusammenhang mit Professionalität und Professionalisierung nehmen im Kontext der Lehrer*innenbildung eine prominente Rolle ein - auch in der Religionslehrer*innenbildung. Die Tatsache, dass an einigen Schulen konfessioneller islamischer Religionsunterricht unterrichtet wird, obwohl es für solche Lehrkräfte, die dieses Fach unterrichten (sollen), an Ausbildungsmöglichkeiten mangelt, macht diese Fragen mit Bezug auf islamische Religionslehrkräfte besonders virulent.

Der Autor leistet durch die Thematisierung der Professionalisierung islamischer Religionslehrkräfte und durch die Genese gehaltvollen Materials, das die Perspektive der Lehrkräfte spiegelt, somit langfristig einen sehr wichtigen Beitrag zur Qualitätssicherung des (islamischen) Religionsunterrichts sowie zur Sensibilisierung für die Stellung muslimischer Lehrkräfte an den Schulen. Tuna trägt weiterhin zur Beantwortung der Forschungsfrage bei, welche Einflussfaktoren auf die Lehrkräfteprofessionalisierung sich bestimmen lassen und deckt die Wahrnehmung von islamischen Religionslehrkräften hinsichtlich ihrer Berufsentwicklung und des Lehrkraft-Seins auf.

Ausgangspunkt dafür markieren narrativ-problemzentrierte Interviews, die durch eine Situationsanalyse das Herausarbeiten von solchen Faktoren ermöglichen, die die professionalitätsorientierte Konzeptualisierung bzw. Professionalisierung des Berufes kennzeichnen. Dabei fällt der Blick auf berufliche Werdegänge, Erfahrungen, Erlebnisse, Sichtweisen sowie auf Haltungen und Einstellungen islamischer Religionslehrkräfte. Tuna benennt das Anliegen, das mit der Studie verfolgt wird, deutlich: zur religionspädagogischen Forschung einen Beitrag zu leisten, hinsichtlich der ein Aufholbedarf - nicht zuletzt wegen der von ihm gut beschriebenen Rahmenbedingungen - besteht. Zudem verfolgt er das Ziel, Einblicke in die Aus-, Fort- und Weiterbildung von islamischen Lehrkräften zu gewähren und deren Rollen- und Berufsverständnis zu erkunden. Der Autor benennt nachstehende Forschungsfragen (25): Welche subjektiv-biografischen Konzepte kennzeichnen islamische Religionslehrkräfte hinsichtlich ihrer Rolle und welche Berufsauffassung haben sie? Was sind in den Augen der Religionslehrkräfte die größten Herausforderungen für die Professionalität? In welchen Wissens- und Kompetenzbereichen halten islamische Religionslehrkräfte eine Ergänzung ihrer Kompetenzen für notwendig?

In einem ersten Kapitel führt der Autor ein, indem er islamische Religionslehrkräfte im Wandel thematisiert und die Rolle beschreibt, die Religion „in der Geschichte des Lehrerberufs und in der Entwicklung des Schulsystems“ (13) einnimmt. Das Kapitel leitet zur Problemstellung hin und kann angesichts der eigenen empirischen Studie nicht im Fokus stehen, auch wenn detailliertere Ausführungen insbesondere für diejenigen wünschenswert wären, die sich bisher kaum mit der islamischen Religionslehrkräfteprofessionalisierung auseinandergesetzt haben. In Kapitel zwei wird die Entwicklung des islamischen Religionsunterrichts und der Religionslehrkräfte, die diesen erteilen, beschrieben. Dabei liegt der Fokus auf Entwicklungen in Österreich. Festgehalten wird, dass die Ausbildung islamischer Religionslehrkräfte nicht losgelöst ist von der - und zugleich notwendig für die - wissenschaftliche(n) Auseinandersetzung mit dem islamischen Religionsunterricht und der Religionslehrkräftebildung (23). Kapitel drei dient der Darstellung des Forschungsstandes und gliedert sich in Ausführungen zum islamischen Religionsunterricht und zu islamischen Religionslehrkräften. Dabei fällt auf, dass es wenig wissenschaftliche Befunde gibt (30). Interessant wäre an dieser Stelle eine Bezugnahme auf Forschungsergebnisse, die sich auf die Professionalität und Professionalisierung von katholischen und evangelischen Religionslehrkräften und Lehrkräften verwandter Fächer (z. B. Philosophie, Geschichte) beziehen sowie der Rückgriff auf Befunde aus dem allgemeinen bzw. erziehungswissenschaftlichen Diskurs zur Lehrkräfteprofessionalisierung.

In Kapitel vier werden Begriffe eingeführt (z. B. Profession und Professionalität, Professionalisierung und Professi- 
onsentwicklung), Zugänge zur Professionsforschung dargestellt und Wege der Professionalisierung und Professionalität aufgezeigt. Das Kapitel umfasst 20 Seiten, sodass durch die multiperspektivische Betrachtung die Thematik zwar vielseitig, aber nicht tiefgründig und nicht durch eine intensive Literaturverarbeitung aufgearbeitet werden kann. Einzelne Unterkapitel umfassen daher nur zehn Zeilen (43). Dadurch ergibt sich, dass z. B. die im Resümee getätigte Aussage, „dass die Lehrer*innen-Professionalisierung und -Bildung ein Prozess ist, der zum Großteil am Arbeitsplatz stattfinde und sich daher, wenn er angemessen begleitet wird, dort auch ohne vorausgegangene Begleitung vollziehen kann“ (51f.), angesichts der Befunde zum Lernen am Arbeitsplatz sicher nicht unplausibel erscheint, gleichzeitig die verkürzten Ausführungen jedoch die Herleitung, das Verständnis und die Bewertung dieser Aussage erschweren.

Kapitel fünf widmet sich dem methodischen Vorgehen. Gelungen ist die Transparenz bezogen auf die Wahl des methodischen Vorgehens. Die Anliegen der Situationsanalyse werden gut beschrieben und zur Grounded Theory in Beziehung gesetzt. Zudem ist die methodische Konzeptualisierung präzise erklärt. Das sechste Kapitel ist das umfangreichste (80-244) und widmet sich der Ergebnisdarstellung. Die Kategorien werden eingeführt und ihnen werden nachvollziehbare Subkategorien zugeordnet. Das Verhältnis von Kategorie und Subkategorien wird durch verständliche Abbildungen visualisiert. Das Ergebniskapitel umfasst spannende und gleichermaßen bedenkenswerte Befunde. Der Autor illustriert seine Ausführungen durch zahlreiche Zitate aus den Interviews. Diese ermöglichen einen authentischen Blick auf die Perspektive der islamischen Religionslehrkräfte.

Nach der Ergebnisdarstellung werden neun Thesen formuliert, die auf den Ergebnissen der eigenen Studie gründen. Nachdem der Autor die Thesen darlegt, werden diese diskutiert. In Kapitel acht werden die Ergebnisse „im Lichte relevanter wissenschaftlicher Diskurse“ (259) verortet, wobei Bezug auf unterschiedliche wissenschaftliche Perspektiven genommen wird. Das Buch endet mit einem kurzen Fazit (Kap. 9).

Insgesamt ist auffällig, dass viele Begriffe, die in Kapitel sechs, sieben und acht verwendet werden (u. a. Motivation, Learning by Doing, Resilienz) zuvor nicht definiert werden bzw. ihre Relevanz für die Frage nach Professionalität und Professionalisierung nicht erläutert wird und die theoretischen Rahmungen (z. B. Professioneller religionspädagogischer Habitus, Faktoren der Professionalisierung) erst in Kapitel acht und somit nach der Ergebnispräsentation stehen. Dadurch wird nicht die gesamte Studie theoretisch gerahmt, sondern lediglich die Ergebnisse. Anzumerken ist hinzu, dass die ersten drei Kapitel sehr kurz sind. Untypisch erscheint auf den ersten Blick dabei, dass die Forschungsfragen hergeleitet werden, bevor Begriffe definiert werden und der Forschungsstand dargestellt wird. Schade ist, dass das Inhaltsverzeichnis lediglich ermöglicht, Kapitel auf der ersten und zweiten Gliederungsebene zu finden. Insgesamt besticht die Arbeit von Tuna jedoch durch den explorativen Charakter und die Fülle an interessanten und authentischen Ergebnissen, die die Perspektive islamischer Religionslehrkräfte wiedergeben. 